Article

\title{
Preparation and Solid-State Characterization of Eltrombopag Crystal Phases
}

\author{
Vincenzo Mirco Abbinante ${ }^{1}$, Massimo Zampieri ${ }^{2, *}$, Giuseppe Barreca ${ }^{2}$ and Norberto Masciocchi ${ }^{1, *}$ (D) \\ 1 Dipartimento di Scienza e Alta Tecnologia \& To.Sca.Lab., Università dell’Insubria, via Valleggio 11, \\ 22100 Como, Italy; vmabbinante@uninsubria.it \\ 2 Chemessentia srl, via Bovio 6, 28100 Novara, Italy; giuseppe.barreca@chemessentia.it \\ * Correspondence: massimo.zampieri@chemessentia.it (M.Z.); norberto.masciocchi@uninsubria.it (N.M.); \\ Tel.: +39-0321-650-255 (M.Z.); +39-031-238-6613 (N.M.)
}

check for

updates

Citation: Abbinante, V.M.; Zampieri,

M.; Barreca, G.; Masciocchi, N.

Preparation and Solid-State

Characterization of Eltrombopag

Crystal Phases. Molecules 2021, 26, 65.

https://dx.doi.org/10.3390/

molecules26010065

Academic Editor: Carlos Eduardo Sabino Bernardes

Received: 9 December 2020

Accepted: 22 December 2020

Published: 25 December 2020

Publisher's Note: MDPI stays neutral with regard to jurisdictional claims in published maps and institutional affiliations.

Copyright: () 2020 by the authors. Licensee MDPI, Basel, Switzerland. This article is an open access article distributed under the terms and conditions of the Creative Commons Attribution (CC BY) license (https: / / creativecommons.org/ licenses/by/4.0/).

\begin{abstract}
Eltrombopag, of $\mathrm{C}_{25} \mathrm{H}_{22} \mathrm{~N}_{4} \mathrm{O}_{4}$ chemical formula, is a drug used against thrombocytopenia, marketed worldwide under different tradenames in the form of its bis-olamine salt. The free acid (CAS no. 496775-61-2) is an intermediate species used for the final drug isolation and is reported to crystallize in more than 20 distinct crystal forms, including a large number of hydrates and solvates. Their identification, and, ultimately, their quantification in industrial lots require the usage of accurately measured X-ray powder diffraction pattern, as well as the assessment of the metrical features (crystal symmetry and lattice parameters), nowadays accessible by powerful crystallographic software. Here, the complete indexing of 13 monophasic samples, prepared using literature or newly tailored crystallization methods, jointly to simultaneous thermogravimetric and calorimetric analyses and to variable temperature $\mathrm{X}$-ray diffraction studies, provide a clear picture of the stability fields of the different crystal phases and their mutual interconversion processes, leading, in a few cases, to new and unexpected crystalline polymorphs or solvates of the pristine unsolvated Form I.
\end{abstract}

Keywords: Eltrombopag; polymorphism; X-ray diffraction; thermal analysis; pattern indexing

\section{Introduction}

Eltrombopag, or 3'-\{(2Z)-2-[1-(3,4-dimethylphenyl)-3-methyl-5-oxo-1,5-dihydro-4Hpyrazol-4-yliden] hydrazino\}-2' hydroxy-3- biphenylcarboxylic acid (CAS no. 496775-61-2), is a drug jointly discovered in 2006 by GlaxoSmithKline and Ligand Pharmaceuticals [1] that has been used, since its U.S. Food and Drug Administration (FDA)approval in 2008 [2], against thrombocytopenia, which is paucity of platelets in blood (thrombocytes). The conventional sketch of the molecular connectivity is shown in Scheme 1.<smiles>Cc1ccc(-n2[nH]c(C)c(N/N=C3/C=CC=C(c4cccc(C(=O)O)c4)C3=O)c2=O)cc1C</smiles>

Scheme 1. Sketch of the Eltrombopag molecular connectivity. 
Eltrombopag is nowadays marketed in the USA under the tradename of Promacta and, in the EU, as Revolade (in both cases, by Novartis). More local brand names include Elbonix, Treptora, ETP, Eltopag, and Cytopag (mostly in southern Asiatic countries). The most common formulation is the bisolamine salt, which was found to be easy to produce, environmentally stable, and easily ingestible from oral suspensions [3]. Thus, the free acid is not commercialized per se, but is the starting material for the obtention of the marketed active pharmaceutical ingredient (API) that is obtained after precipitation from a methanolic solution with excess 2-aminoethanol [4].

APIs often exist in different solid-state forms, either crystalline or amorphous, and may be isolated as pure systems, or in hydrates, solvates, salts, and cocrystals [5,6]. Each solid form, including polymorphic ones, possesses its own molecular arrangement, dictated by distinct intermolecular interactions and conformationally driven crystal packing, leading to remarkably different physico-chemical properties. Consequently, melting points, crystal polarity and chirality, solubility, thermal and chemical stability (or inertness), crystal size and size distribution may show distinct features; these differences are further augmented by other effects typical of anisotropic media, such as shape of the crystals, preferential cleavage, hardness, compressibility and flowability [7-9]. These effects are particularly relevant during synthesis, isolation, and purification processes, as well as in the formulation steps (where rheological parameters become important during drug transport and tableting). Hygroscopicity and particle size and shape distributions may also affect shelf life of the marketed drug, and, more importantly, also pharmacokinetic and bioavailability properties may be dramatically changed, making the drug useless or even toxic [10].

As with many other cases in the vast literature on solid state forms of API, Eltrombopag also manifests the presence of several truly polymorphic (unsolvated) crystal phases, and, depending on the type of solvent from which is it recovered, also manifests many solvated phases containing different stoichiometric quantities of the solvent molecules in the crystal lattice [11-13]. To assist the reader in interpreting the large number of results obtained on this series of polymorphs and solvates, we have provided the correct assignments of the different species (or mixture thereof) at the end of this paper, clearing, to some extent, inexact or contradicting observations from the literature.

As Eltrombopag (free acid) is not the final, commonly supplied form to the patients, some of these issues, which remain valid for the Eltrombopag bisolamine system, vanish; however, knowledge of the crystal chemistry and the thermal stability and evolution paths of the many forms claimed in the patent literature (collectively listed in Table 1) is a must, as well as understanding the possible occurrence of polyphasic mixtures, (mis)taken, in the absence of a complete (at least metrical) characterization, for a single form.

This paper, therefore, revises the zoo of Eltrombopag free acid polymorphs and solvates; tries to determine phase purity by conventional powder pattern indexing; it also provides, through variable-temperature in situ X-ray powder diffraction (XRPD) experiments, clues on the thermal stability and thermally induced evolution/desolvation of the Eltrombopag crystal phases which, not being isolated in milligram quantities within a Differential Scanning Calorimetry (DSC)apparatus, can be ideally prepared within pilot or industrial plants. More importantly, the complete list of the certified peak positions and relative intensities, obtained from crystallographic analysis, can be safely used for identifying a specific crystal phase, also if present in very minor quantities (say, below $1 \mathrm{wt} \%$ level), if accurate XRPD measurements are taken. 
Table 1. Known Eltrombopag free acid polymorphs and solvates described in the literature.

\begin{tabular}{cccc}
\hline Form Name & Nature & Reference & Comments \\
\hline I & Crystalline, unsolvated & {$[11]$} & Reprepared \\
II & Crystalline, bis-ethanolamine salt & {$[11]$} & Not relevant (salt) \\
III & Crystalline, hydrate & {$[11]$} & Form XIV obtained \\
IV & Crystalline, not specified & {$[11]$} & Form I obtained \\
V & Crystalline, THF/water solvate & {$[11]$} & Reprepared \\
VI & Crystalline, not specified & {$[11]$} & Reprepared \\
VII & Crystalline, not specified & {$[11]$} & Reprepared \\
VIII & Crystalline, not specified & {$[11]$} & Not obtained \\
IX & Crystalline, not specified & {$[11]$} & Reprepared \\
X & Crystalline, not specified & {$[11]$} & Reprepared \\
XI & Crystalline, not specified & {$[11]$} & Reprepared \\
XII & Crystalline, not specified & {$[11]$} & Reprepared \\
XIII & Crystalline, not specified & {$[11]$} & Not obtained \\
XIV & Crystalline, not specified & {$[11]$} & Reprepared \\
XV & Crystalline, not specified & {$[11]$} & Not attempted (mixture) \\
XVI & Crystalline, monohydrate & {$[11]$} & Synthesis not attempted \\
Z & Crystalline, not specified & {$[12]$} & Equal to DMF solvate \\
Amorphous & Fully amorphous & {$[12]$} & Not relevant (amorphous) \\
DMF solvate & Crystalline, DMF solvate & {$[12]$} & Reprepared \\
DMA solvate & Crystalline, DMA solvate & {$[12]$} & Reprepared \\
NMP solvate & Crystalline, NMP solvate & {$[12]$} & Reprepared \\
H1 & Crystalline, not specified & {$[13]$} & Equal to DMF solvate \\
\hline
\end{tabular}

\section{Results and Discussion}

\subsection{Preliminary Considerations}

Form II was not reprepared, as it is not an Eltrombopag free acid (alone, or solvated) crystal phase, but, instead, a bis-ethanolamine salt, i.e., the marketed active pharmaceutical ingredient. Attempts to prepare Form III and Form IV, following the published procedures, did not result into the sought materials but, instead, lead to the isolation of Form XIV and Form I, respectively, later discussed. The unavailability of form IV impeded the preparation of Form VIII, which was derived by heating Form IV [11]. The preparation of Form XIII (in diethyl-ether) also failed and was attributed to the very scarce solubility of Form I, even at reflux conditions. Comparison of published data indicated that Form XV is a mixture of forms $X$ and $X a$ (now prepared independently as pure crystal forms) and that Forms $\mathrm{Z}$ and $\mathrm{H} 1$ match the $\mathrm{N}, \mathrm{N}$-dimethylformamide (DMF) solvate form. The synthesis of Form XVI was not attempted, as it precipitated during the direct condensation of 3'-amino-2'-hydroxybiphenyl-3-carboxylix acid and 1-(3,4-dimethylphenyl)-3-methyl-1Hpyrazol-5-ol [11], not available in our laboratory. Finally, the amorphous material reported in [12], falling out of the scope of this study, was not considered.

All the crystal phases, whether unsolvated polymorphs or solvates of Etrombopag free acid, were characterized, in primis, by XRPD, which was used to assess phase purity and, after indexing, the unit cell size and shape, and ultimately their symmetry (crystal system and space group). As the raw data can be fruitfully used for fingerprinting, we propose in separate figures comparative plots of the unsolvated and solvated phases (Figures 1-6); these figures contain the low-angle portions of the raw X-ray diffraction data, i.e., those that are normally used for identification purposes, as well for detecting the presence of contaminant phases. Tables $2-14$ contain the observed $2 \theta$ angles, $\mathrm{d}$-spacings, and relative intensities of the prominent indexed diffraction peaks (used in the cell determination procedure) and are reported within each pertinent paragraph. Comparative lists of our results and of the relevant crystal data, which include all determined unit cells, are shown in Tables 15 and 16. 


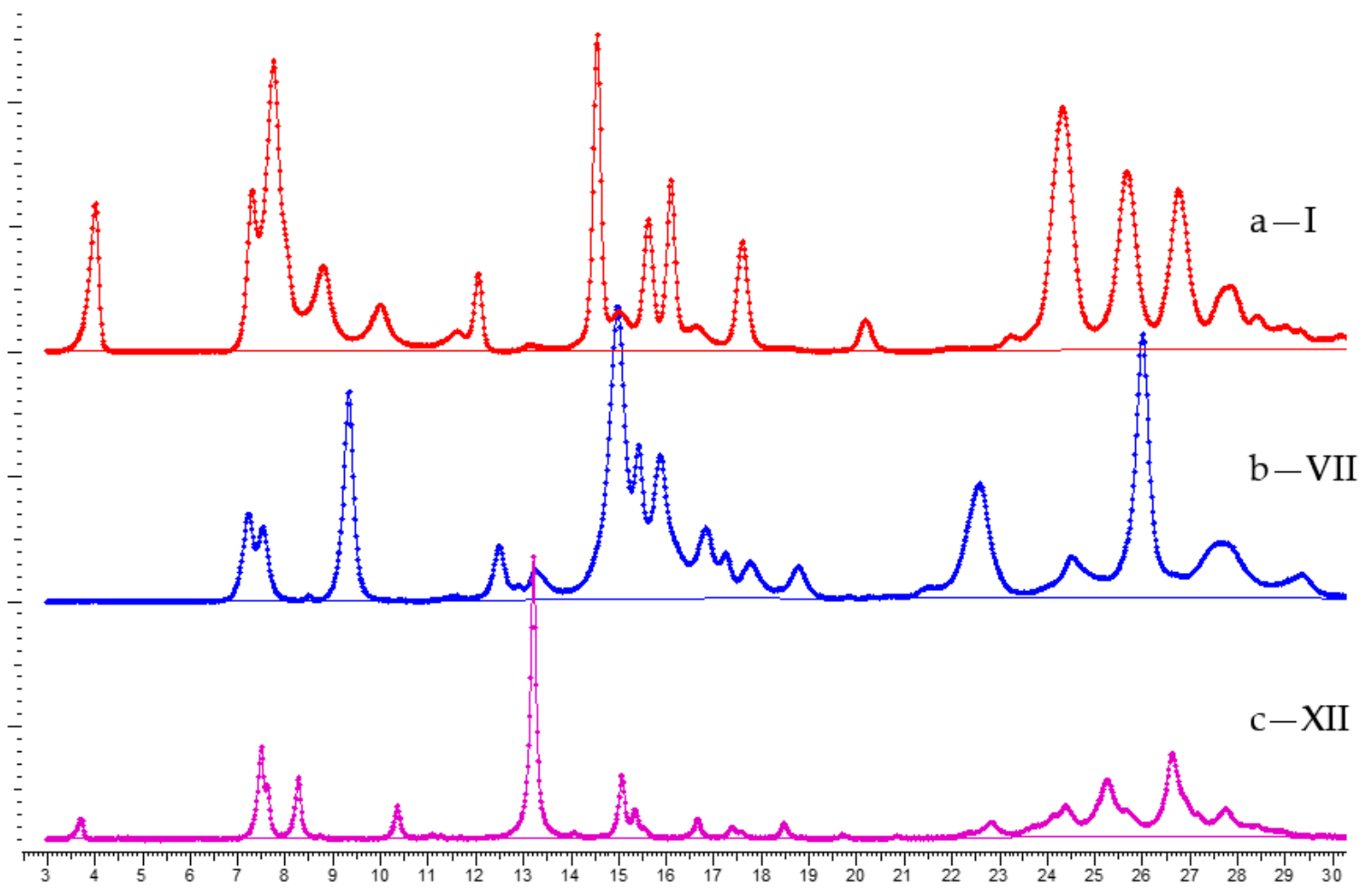

Figure 1. Raw X-ray powder diffraction (XRPD) data for unsolvated Eltrombopag free acid polymorphs ((a) Form I; (b) Form VII, and (c) Form XII), in the low-angle $\left(3-30^{\circ} 2 \theta\right)$ range. $x$ and y axes: $2 \theta$ range, ${ }^{\circ}$ and intensities (a.u.), respectively. These raw data clearly demonstrate the higher crystallinity (narrower Bragg peaks) of Form XII (c) than for Form I (a) and Form VII (b).

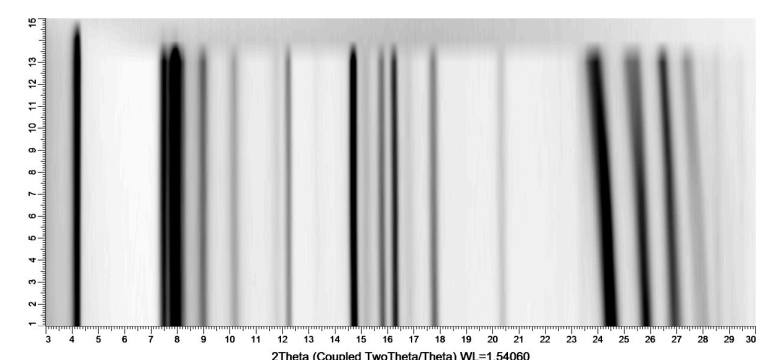

(a) - Form I

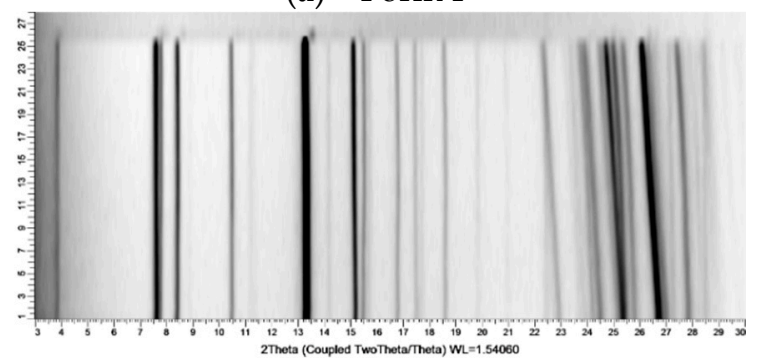

(c) -Form XII

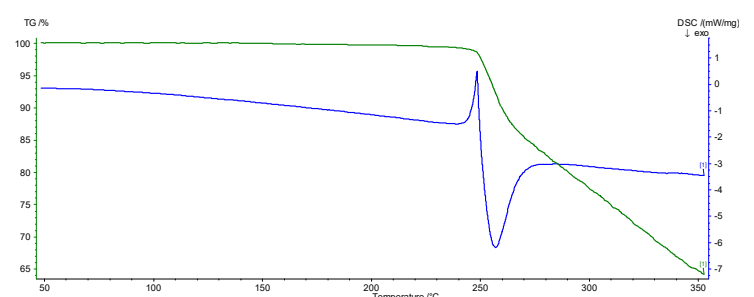

(b)

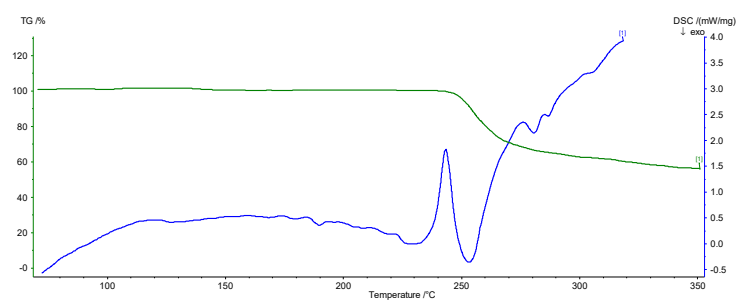

(d)

Figure 2. Variable Temperature X-ray Diffraction (VTXRD) traces of Form I (a) and Form XII (c). Horizontal scale: $2 \theta\left({ }^{\circ}\right)$; vertical scale: progressive number of VTXRD measurements in isothermal conditions (in $20^{\circ} \mathrm{C}$ steps each, in the $30-270{ }^{\circ} \mathrm{C}$ range). In panels (b) and (d), the pertinent TG (in green) and DSC (in blue) traces show that the unique thermally induced event, in both Form I and Form XII, is the incipient melting/decomposition occurring near $250{ }^{\circ} \mathrm{C}$. 


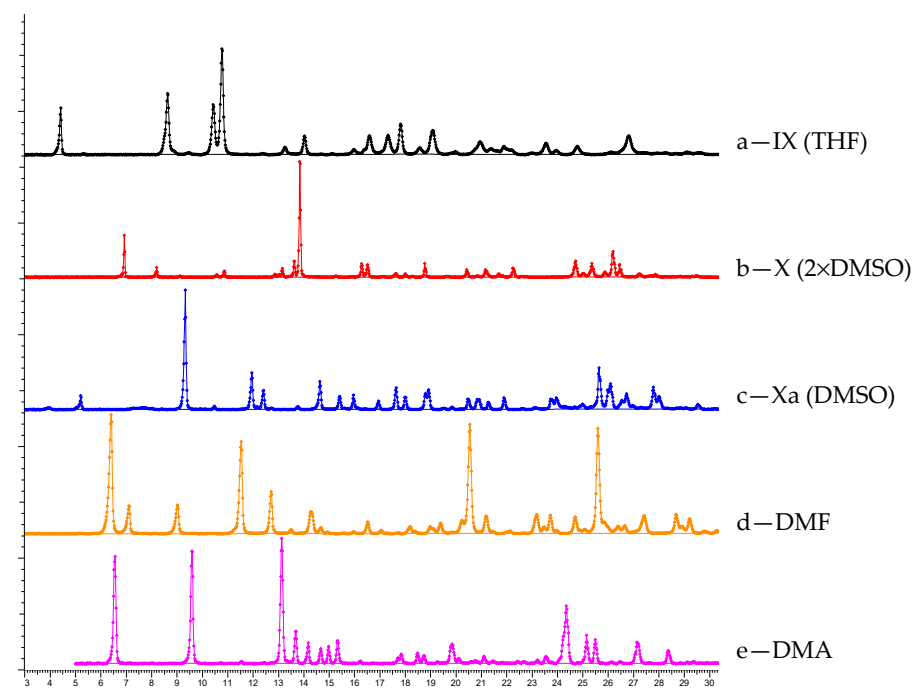

Figure 3. Raw XRPD data for solvated Eltrombopag free acid species: (a) Form IX; (b) Form X; (c) Form Xa; (d) DMF solvate; and (e) DMA solvate, in the low-angle ( $3-30^{\circ} 2 \theta$ ) range. $x$ and y axes: $2 \theta$ range, ${ }^{\circ}$ and intensities (a.u.), respectively. These raw data clearly manifested the high crystallinity (narrow Bragg peaks) of all these stoichiometric solvates.

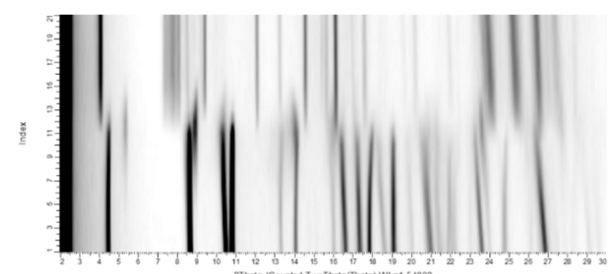

(a)-Form IX

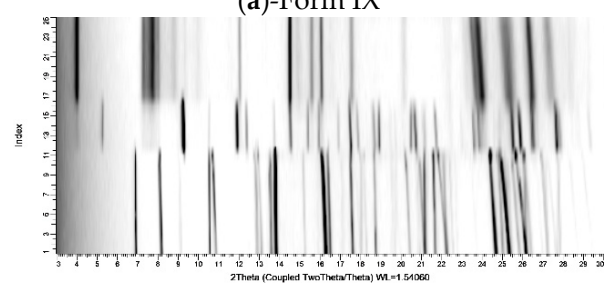

(c)-Form X

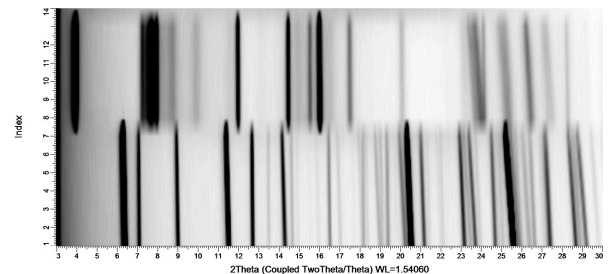

(e)-DMF solvate

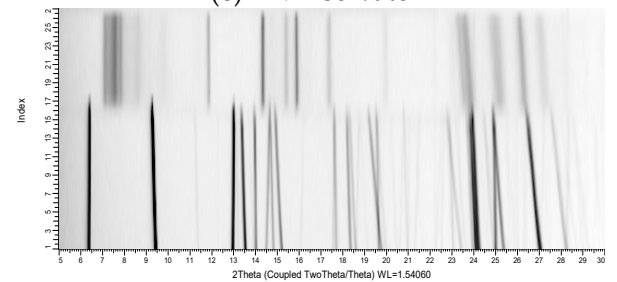

(g)-DMA solvate

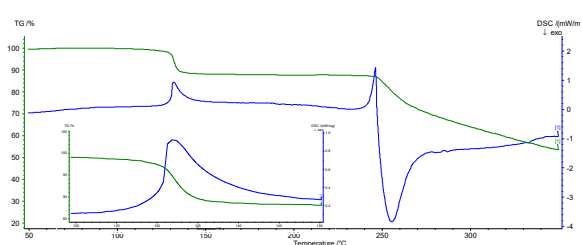

(b)

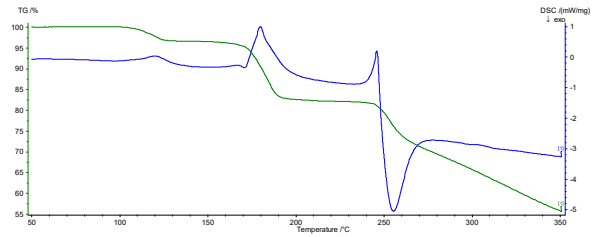

(d)

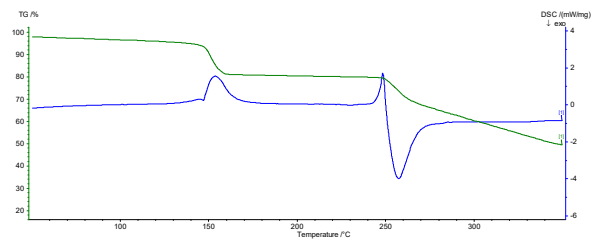

(f)

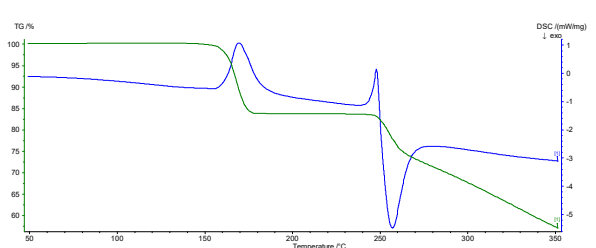

(h)

Figure 4. VTXRD of Form IX (a), Form X (c), DMF solvate (e), and DMA solvate (g). Horizontal scale: $2 \theta\left(^{\circ}\right)$; vertical scale: progressive number of VTXRD measurement in isothermal conditions (in $20^{\circ} \mathrm{C}$ steps each, in the $30-240{ }^{\circ} \mathrm{C}$ range). In panels (b,d,f,h), the pertinent TG (in green) and DSC (in blue) traces showing the thermally induced events, the intermediacy of the IXa and Xa phases discussed in the text, and the quantitative formation at high enough temperatures of Form I (decomposing near $250^{\circ} \mathrm{C}$ ). In panel (b), the inset shows the relevant high-T tail of the weak endotherm associated to the formation of Form IXa, discussed in the text. 


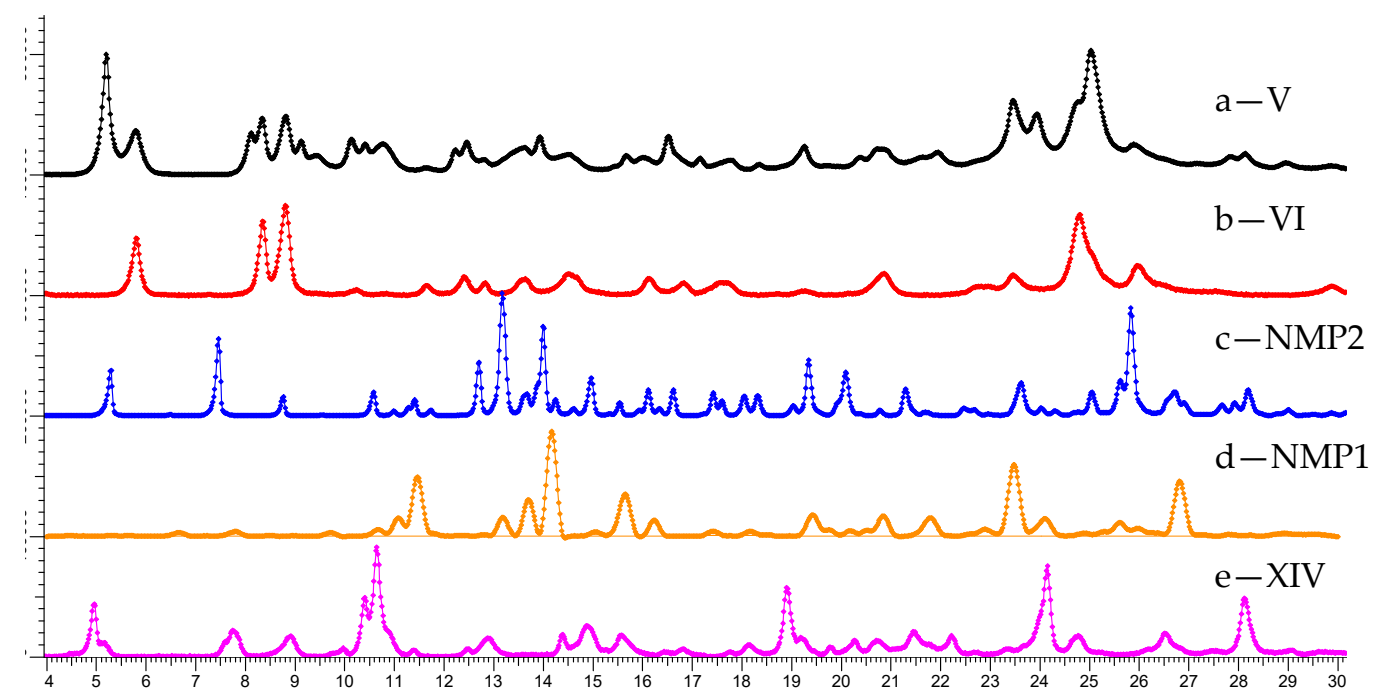

Figure 5. Raw XRPD data for solvated Eltrombopag free acid species: (a) Form V; (b) Form VI; (c) Form NMP (2:1) solvate; (d) NMP (1:1) solvate; and (e) Form XIV in the low-angle (4-30 $2 \theta$ ) range. $x$ and y axes: $2 \theta$ range, ${ }^{\circ}$ and intensities (a.u.), respectively. These raw data clearly manifested the high crystallinity (narrow Bragg peaks) of the NMP2 crystal phase, while broader peaks were observed for all other solvates.

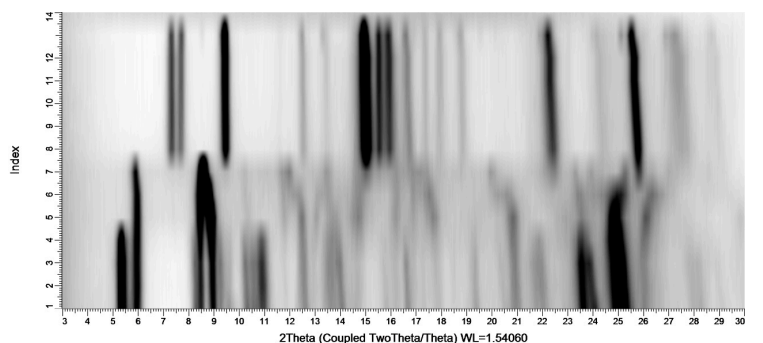

(a)-Form V

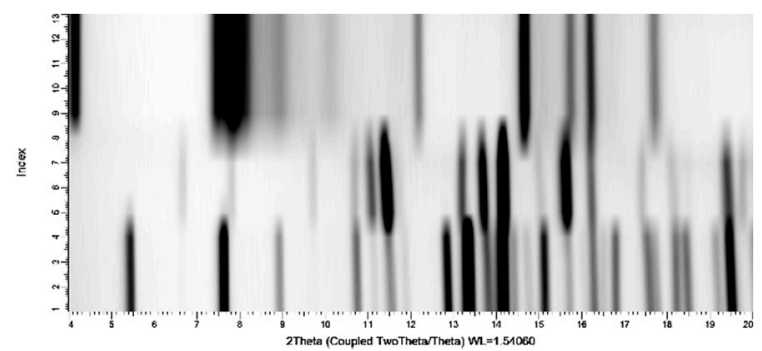

(c)-NMP Solvates

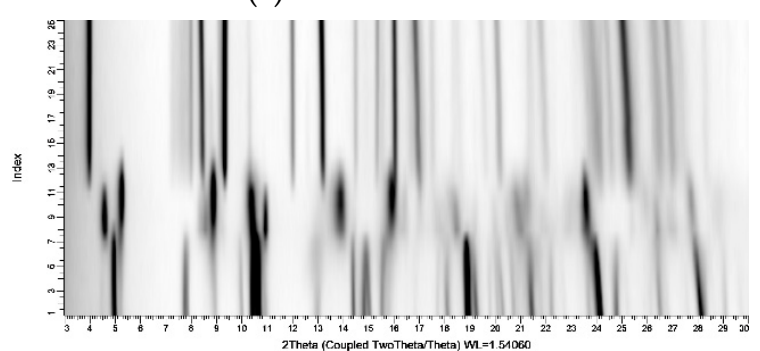

(e)-Form XIV

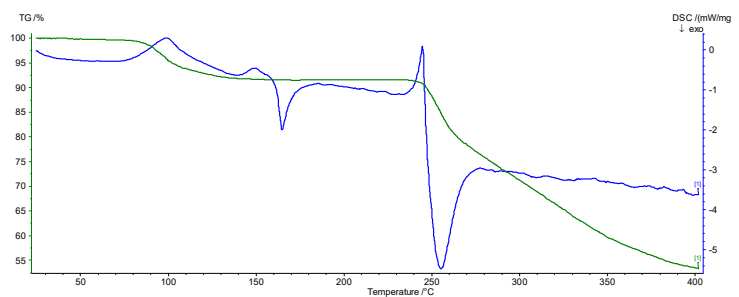

(b)

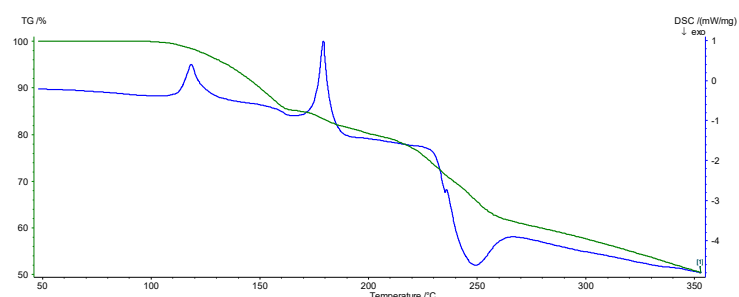

(d)

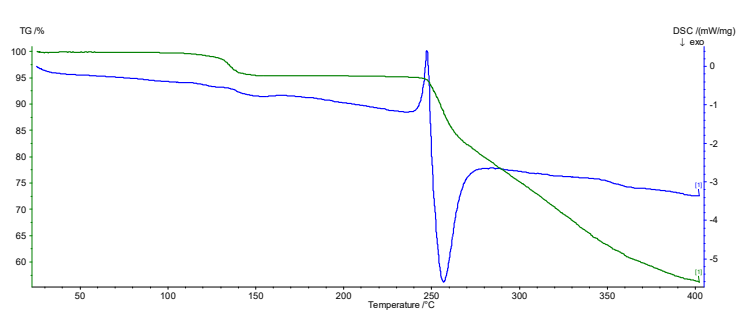

(f)

Figure 6. VTXRD and TG/DSC traces of Form V $(\mathbf{a}, \mathbf{b})$, the original NMP2-rich mixture of NMP1 and NMP2 crystal phases $(\mathbf{c}, \mathbf{d})$, and Form XIV $(\mathbf{e}, \mathbf{f})$. Horizontal scale: $2 \theta\left(^{\circ}\right)$; vertical scale: progressive number of VTXRD measurements in isothermal conditions (in $20^{\circ} \mathrm{C}$ steps each, typically in the $30-240{ }^{\circ} \mathrm{C}$ range). 
Table 2. List of observed $2 \theta$ angles, $d$-spacings, and relative intensities, jointly with reflection indices, for Eltrombopag Form I.

\begin{tabular}{cccc}
\hline 20 Angle, & d-Spacing, $\mathbf{A}^{\circ}$ & $\mathbf{I}_{\text {rel }}$ & h k $\mathbf{~}$ \\
\hline 3.999 & 22.074 & 50 & 001 \\
7.278 & 12.135 & 52 & 010 \\
7.761 & 11.380 & 93 & $0-11$ \\
7.989 & 11.057 & 37 & 002 \\
8.794 & 10.046 & 28 & 011 \\
10.003 & 8.834 & 15 & $0-12$ \\
11.613 & 7.613 & 6 & 012 \\
12.034 & 7.348 & 25 & 003 \\
13.135 & 6.734 & 2 & $0-13$ \\
14.538 & 6.087 & 100 & 020 \\
14.997 & 5.902 & 23 & 013 \\
15.627 & 5.665 & 42 & 021 \\
16.086 & 5.505 & 55 & 004 \\
16.673 & 5.312 & 35 & $0-14$ \\
17.605 & 5.033 & 1 & 022 \\
18.626 & 4.760 & 10 & 014 \\
20.166 & 4.399 & $<1$ & 005 \\
21.418 & 4.145 & 1 & 100 \\
21.922 & 4.051 & 1 & $10-1$ \\
22.512 & 3.946 & & 110 \\
\hline
\end{tabular}

Table 3. List of observed $2 \theta$ angles, d-spacings, and relative intensities, jointly with reflection indices, for Eltrombopag Form VII.

\begin{tabular}{|c|c|c|c|}
\hline $2 \theta$ Angle, $^{\circ}$ & d-Spacing, §̊ & $I_{\text {rel }}$ & h k 1 \\
\hline 7.243 & 12.193 & 28 & 001 \\
\hline 7.530 & 11.729 & 24 & 010 \\
\hline 9.343 & 9.457 & 67 & $0-11$ \\
\hline 11.520 & 7.675 & 2 & 011 \\
\hline 12.501 & 7.074 & 17 & $10-1$ \\
\hline 12.878 & 6.868 & 6 & $1-10$ \\
\hline 13.261 & 6.671 & 10 & $1-1-1$ \\
\hline 14.570 & 6.074 & 16 & 002 \\
\hline 14.982 & 5.908 & 100 & $0-12$ \\
\hline 15.425 & 5.739 & 50 & $0-21$ \\
\hline 15.880 & 5.576 & 46 & $11-1$ \\
\hline 16.825 & 5.265 & 23 & 101 \\
\hline 17.256 & 5.134 & 16 & $1-1-2$ \\
\hline 17.770 & 4.987 & 13 & $1-2-1$ \\
\hline 18.787 & 4.719 & 11 & $0-22$ \\
\hline 21.442 & 4.140 & 4 & $1-2-2$ \\
\hline 22.566 & 3.936 & 38 & $1-30$ \\
\hline 24.073 & 3.693 & 5 & $0-23$ \\
\hline 24.516 & 3.628 & 14 & $20-1$ \\
\hline 24.827 & 3.583 & 10 & $2-1-2$ \\
\hline
\end{tabular}


Table 4. List of observed $2 \theta$ angles, $d$-spacings, and relative intensities, jointly with reflection indices, for Eltrombopag Form XII.

\begin{tabular}{|c|c|c|c|}
\hline $2 \theta$ Angle, ${ }^{\circ}$ & d-Spacing, Å & $\mathbf{I}_{\text {rel }}$ & h k 1 \\
\hline 3.722 & 23.715 & 7 & 001 \\
\hline 7.513 & 11.757 & 33 & 002 \\
\hline 7.631 & 11.575 & 19 & 010 \\
\hline 8.294 & 10.650 & 22 & 011 \\
\hline 8.740 & 10.109 & 1 & $0-11$ \\
\hline 10.374 & 8.520 & 11 & 012 \\
\hline 11.100 & 7.964 & 2 & $0-12$ \\
\hline 11.280 & 7.838 & 1 & 003 \\
\hline 13.226 & 6.688 & 100 & 013 \\
\hline 14.109 & 6.271 & 2 & $0-13$ \\
\hline 15.075 & 5.872 & 22 & 004 \\
\hline 15.358 & 5.764 & 10 & 020 \\
\hline 15.544 & 5.695 & 4 & 021 \\
\hline 16.641 & 5.322 & 7 & 022 \\
\hline 17.386 & 5.096 & 4 & $0-14$ \\
\hline 17.552 & 5.048 & 3 & $0-22$ \\
\hline 18.490 & 4.794 & 5 & 023 \\
\hline 18.904 & 4.690 & $<1$ & 005 \\
\hline 19.704 & 4.501 & 2 & $0-23$ \\
\hline 20.877 & 4.251 & 1 & 024 \\
\hline 22.346 & 3.975 & 2 & $0-24$ \\
\hline 22.829 & 3.892 & 6 & 006 \\
\hline 23.720 & 3.748 & 4 & 025 \\
\hline 24.105 & 3.688 & 8 & 101 \\
\hline 24.388 & 3.646 & 12 & $1-10$ \\
\hline 25.260 & 3.522 & 21 & $11-4$ \\
\hline 25.664 & 3.468 & 10 & $10-5$ \\
\hline 26.623 & 3.345 & 31 & 007 \\
\hline 26.906 & 3.311 & 14 & $12-2$ \\
\hline 27.182 & 3.278 & 9 & 120 \\
\hline 27.760 & 3.211 & 11 & $10-6$ \\
\hline
\end{tabular}

Table 5. List of observed $2 \theta$ angles, $d$-spacings, and relative intensities, jointly with reflection indices, for Eltrombopag Form IX.

\begin{tabular}{|c|c|c|c|}
\hline $2 \theta$ Angle, $^{\circ}$ & d-Spacing, Å & $\mathbf{I}_{\text {rel }}$ & h k 1 \\
\hline 4.415 & 19.997 & 42 & 001 \\
\hline 8.481 & 10.417 & 55 & 010 \\
\hline 8.625 & 10.243 & 4 & $0-11$ \\
\hline 10.438 & 8.468 & 44 & 011 \\
\hline 10.756 & 8.217 & 100 & $0-12$ \\
\hline 13.256 & 6.673 & 6 & 003 \\
\hline 14.011 & 6.315 & 16 & $0-13$ \\
\hline 14.677 & 6.030 & $<1$ & $10-1$ \\
\hline 15.101 & 5.861 & $<1$ & 101 \\
\hline 15.968 & 5.545 & 4 & $1-11$ \\
\hline 16.370 & 5.410 & 4 & $10-2$ \\
\hline 16.581 & 5.342 & 17 & $0-21$ \\
\hline 17.306 & 5.119 & 17 & $11-1$ \\
\hline 17.800 & 4.978 & 27 & $0-14$ \\
\hline 18.571 & 4.773 & 6 & 021 \\
\hline 19.059 & 4.652 & 22 & $0-23$ \\
\hline 19.817 & 4.476 & 1 & $1-13$ \\
\hline 20.002 & 4.435 & 2 & 103 \\
\hline 20.769 & 4.273 & 5 & 112 \\
\hline 20.970 & 4.232 & 12 & 022 \\
\hline
\end{tabular}


Table 6. List of observed $2 \theta$ angles, $d$-spacings, and relative intensities, jointly with reflection indices, for Eltrombopag Form X.

\begin{tabular}{cccc}
\hline 20 Angle, & d-Spacing, ${ }^{\circ}$ & $\mathbf{I}_{\text {rel }}$ & h k $\mathbf{~}$ \\
\hline 6.927 & 12.749 & 36 & 001 \\
8.210 & 10.760 & 8 & 010 \\
8.740 & 10.109 & $<1$ & 101 \\
9.135 & 9.672 & 1 & 100 \\
10.571 & 8.361 & 2 & 011 \\
10.878 & 8.126 & 5 & $0-11$ \\
12.870 & 6.872 & 2 & 102 \\
13.035 & 6.786 & 2 & $1-11$ \\
13.159 & 6.722 & 8 & $1-10$ \\
13.631 & 6.491 & 14 & $10-1$ \\
13.851 & 6.388 & 100 & 002 \\
15.279 & 5.794 & 1 & $11-1$ \\
15.920 & 5.562 & $<1$ & 012 \\
16.284 & 5.438 & 12 & $0-12$ \\
16.522 & 5.360 & 11 & $1-1-1$ \\
17.164 & 5.162 & $<1$ & 121 \\
17.640 & 5.023 & 3 & 021 \\
18.009 & 4.921 & 3 & $0-21$ \\
18.282 & 4.848 & 1 & 200 \\
18.759 & 4.726 & 12 & 103 \\
\hline
\end{tabular}

Table 7. List of observed $2 \theta$ angles, d-spacings, and relative intensities, jointly with reflection indices, for Eltrombopag Form Xa.

\begin{tabular}{cccc}
\hline 20 Angle, & d-Spacing, $\mathbf{A}$ & $\mathbf{I}_{\text {rel }}$ & $\mathbf{h ~ k ~} \mathbf{~}$ \\
\hline 5.212 & 16.941 & 11 & 001 \\
9.338 & 9.462 & 100 & 200 \\
10.482 & 8.432 & 2 & 002 \\
11.910 & 7.424 & 15 & $20-1$ \\
11.954 & 7.397 & 28 & 202 \\
12.166 & 7.269 & 2 & 110 \\
12.411 & 7.125 & 15 & 011 \\
12.737 & 6.944 & 1 & 111 \\
13.778 & 6.422 & 2 & $1-1-1$ \\
14.635 & 6.047 & 22 & 210 \\
15.418 & 5.742 & 10 & 012 \\
15.961 & 5.548 & 11 & $20-2$ \\
16.944 & 5.228 & 7 & $1-1-2$ \\
17.642 & 5.023 & 17 & 311 \\
18.007 & 4.921 & 10 & 401 \\
18.808 & 4.714 & 12 & 402 \\
18.928 & 4.684 & 15 & 113 \\
19.522 & 4.543 & 1 & $2-1-2$ \\
19.840 & 4.471 & 2 & $3-1-1$ \\
20.488 & 4.331 & 8 & $20-3$ \\
\hline
\end{tabular}


Table 8. List of observed $2 \theta$ angles, $d$-spacings, and relative intensities, jointly with reflection indices, for Eltrombopag DMF solvate.

\begin{tabular}{cccc}
\hline 20 Angle, & d-Spacing, $\boldsymbol{A}^{\circ}$ & $\mathbf{I}_{\text {rel }}$ & h k $\mathbf{~}$ \\
\hline 6.406 & 13.784 & 100 & 002 \\
7.117 & 12.410 & 24 & 011 \\
9.026 & 9.789 & 24 & 012 \\
11.535 & 7.664 & 4 & 013 \\
12.716 & 6.955 & 75 & 020 \\
13.110 & 6.747 & 35 & 021 \\
13.220 & 6.691 & 3 & 101 \\
13.501 & 6.552 & 3 & -101 \\
14.278 & 6.197 & 18 & 022 \\
14.332 & 6.174 & 15 & 014 \\
14.687 & 6.026 & 5 & 111 \\
14.944 & 5.923 & 1 & $-1-11$ \\
15.590 & 5.679 & 1 & 112 \\
15.986 & 5.539 & 9 & 023 \\
16.534 & 5.357 & 3 & -103 \\
17.056 & 5.194 & $<1$ & 113 \\
17.300 & 5.121 & $<1$ & 015 \\
17.744 & 4.994 & 6 & $-1-13$ \\
18.206 & 4.868 & 2 & 120 \\
18.388 & 4.820 & & 121 \\
\hline
\end{tabular}

Table 9. List of observed $2 \theta$ angles, $d$-spacings, and relative intensities, jointly with reflection indices, for Eltrombopag DMA solvate.

\begin{tabular}{cccc}
\hline 20 Angle, & d-Spacing, $\mathbf{A}$ & $\mathbf{I}_{\text {rel }}$ & h $\mathbf{k} \mathbf{~}$ \\
\hline 6.562 & 13.457 & 85 & 001 \\
9.578 & 9.226 & 89 & 011 \\
11.530 & 7.668 & 1 & 100 \\
12.410 & 7.126 & $<1$ & 101 \\
13.132 & 6.736 & 100 & 002 \\
13.675 & 6.469 & 26 & 020 \\
14.158 & 6.250 & 16 & $1-11$ \\
14.646 & 6.043 & 11 & $0-12$ \\
14.972 & 5.912 & 13 & 012 \\
15.331 & 5.774 & 18 & 021 \\
16.202 & 5.466 & 2 & 102 \\
17.703 & 5.005 & 4 & 112 \\
17.839 & 4.968 & 7 & 120 \\
18.484 & 4.796 & 8 & $1-21$ \\
18.722 & 4.735 & 6 & $10-2$ \\
19.251 & 4.606 & 1 & 022 \\
19.781 & 4.484 & 13 & $11-2$ \\
19.861 & 4.466 & 15 & $1-2-1$ \\
20.107 & 4.412 & 4 & $1-1-2$ \\
\hline
\end{tabular}


Table 10. List of observed $2 \theta$ angles, $\mathrm{d}$-spacings, and relative intensities, jointly with reflection indices, for Eltrombopag Form V.

\begin{tabular}{|c|c|c|c|}
\hline $2 \theta$ Angle, $^{\circ}$ & d-Spacing, Å & $\mathbf{I}_{\text {rel }}$ & h k 1 \\
\hline 5.210 & 16.946 & 100 & 001 \\
\hline 8.128 & 10.869 & 34 & 010 \\
\hline 9.127 & 9.681 & 27 & $0-11$ \\
\hline 10.142 & 8.713 & 29 & 011 \\
\hline 10.417 & 8.485 & 25 & 002 \\
\hline 12.238 & 7.226 & 20 & 100 \\
\hline 12.464 & 7.095 & 26 & $0-12$ \\
\hline 13.947 & 6.344 & 31 & 012 \\
\hline 15.687 & 5.644 & 15 & 003 \\
\hline 16.534 & 5.357 & 31 & $0-21$ \\
\hline 17.162 & 5.162 & 13 & $1-12$ \\
\hline 18.355 & 4.829 & 8 & $0-22$ \\
\hline 19.266 & 4.603 & 23 & $1-21$ \\
\hline 19.750 & 4.491 & 7 & $1-2-1$ \\
\hline 20.378 & 4.354 & 13 & 022 \\
\hline 21.627 & 4.105 & 14 & $0-14$ \\
\hline 21.942 & 4.047 & 18 & 120 \\
\hline 23.465 & 3.788 & 62 & $10-4$ \\
\hline 23.948 & 3.712 & 50 & 023 \\
\hline 24.771 & 3.591 & 60 & $20-1$ \\
\hline 25.037 & 3.553 & 100 & $2-1-1$ \\
\hline
\end{tabular}

Table 11. List of observed $2 \theta$ angles, d-spacings, and relative intensities, jointly with reflection indices, for Eltrombopag Form VI.

\begin{tabular}{cccc}
\hline 20 Angle, & d-Spacing, $^{\circ}$ & $\mathbf{I}_{\text {rel }}$ & h k $\mathbf{~}$ \\
\hline 5.815 & 15.183 & 62 & 001 \\
8.361 & 10.566 & 80 & 010 \\
8.820 & 10.016 & 100 & 011 \\
11.659 & 7.583 & 11 & 002 \\
12.418 & 7.121 & 20 & 012 \\
12.837 & 6.890 & 13 & 100 \\
13.560 & 6.524 & 15 & $10-1$ \\
13.666 & 6.474 & 17 & 110 \\
14.493 & 6.106 & 22 & 111 \\
14.673 & 6.032 & 19 & 101 \\
16.136 & 5.488 & 18 & $0-12$ \\
16.848 & 5.257 & 13 & 020 \\
17.376 & 5.099 & 8 & 112 \\
17.525 & 5.056 & 13 & 003 \\
17.740 & 4.995 & 13 & 022 \\
18.750 & 4.728 & 1 & 120 \\
19.252 & 4.606 & 4 & $0-21$ \\
20.859 & 4.255 & 23 & 023 \\
22.736 & 3.907 & 10 & $1-2-1$ \\
\hline
\end{tabular}


Table 12. List of observed $2 \theta$ angles, $\mathrm{d}$-spacings, and relative intensities, jointly with reflection indices, for Eltrombopag NMP2.

\begin{tabular}{cccc}
\hline 20 Angle, & d-Spacing, $\mathbf{A}^{\circ}$ & $\mathbf{I}_{\text {rel }}$ & h k $\mathbf{~}$ \\
\hline 5.284 & 16.708 & 37 & 002 \\
7.463 & 11.835 & 63 & 011 \\
8.764 & 10.080 & 15 & 012 \\
10.590 & 8.346 & 19 & 013 \\
11.738 & 7.532 & 4 & 102 \\
12.705 & 6.961 & 44 & 014 \\
13.175 & 6.714 & 100 & -102 \\
14.251 & 6.209 & 12 & 021 \\
14.621 & 6.053 & 6 & 113 \\
14.965 & 5.915 & 31 & $-1-12$ \\
15.940 & 5.555 & 4 & 006 \\
16.120 & 5.493 & 21 & 023 \\
16.619 & 5.330 & 18 & -104 \\
17.423 & 5.085 & 12 & 016 \\
17.604 & 5.033 & 16 & 024 \\
18.047 & 4.911 & 16 & 120 \\
18.318 & 4.839 & 8 & 122 \\
19.041 & 4.657 & 46 & 123 \\
19.348 & 4.583 & 11 & 025 \\
19.970 & 4.442 & & 002 \\
\hline
\end{tabular}

Table 13. List of observed $2 \theta$ angles, d-spacings, and relative intensities, jointly with reflection indices, for Eltrombopag NMP1.

\begin{tabular}{|c|c|c|c|}
\hline $2 \theta$ Angle, ${ }^{\circ}$ & d-Spacing, Å & $I_{\text {rel }}$ & h k 1 \\
\hline 6.525 & 13.534 & 3 & 001 \\
\hline 7.656 & 11.536 & 4 & 010 \\
\hline 9.589 & 9.216 & 3 & $0-11$ \\
\hline 10.542 & 8.384 & 1 & 011 \\
\hline 10.941 & 8.079 & 6 & $1-10$ \\
\hline 11.306 & 7.819 & 18 & $1-11$ \\
\hline 11.606 & 7.618 & 53 & 101 \\
\hline 13.057 & 6.774 & 17 & 002 \\
\hline 13.561 & 6.523 & 36 & $10-1$ \\
\hline 14.030 & 6.307 & 100 & $1-1-1$ \\
\hline 14.888 & 5.945 & $<1$ & $1-12$ \\
\hline 15.503 & 5.710 & 37 & $1-20$ \\
\hline 16.081 & 5.507 & 16 & $0-21$ \\
\hline 17.274 & 5.129 & 4 & 021 \\
\hline 17.987 & 4.927 & $<1$ & $1-22$ \\
\hline 18.350 & 4.830 & 4 & $10-2$ \\
\hline 19.266 & 4.603 & 16 & 112 \\
\hline 19.610 & 4.523 & 6 & 003 \\
\hline
\end{tabular}


Table 14. List of observed $2 \theta$ angles, $\mathrm{d}$-spacings, and relative intensities, jointly with reflection indices, for Eltrombopag Form XIV.

\begin{tabular}{cccc}
\hline 20 Angle, & d-Spacing, $^{\mathbf{A}}$ & $\mathbf{I}_{\text {rel }}$ & h k l \\
\hline 4.959 & 17.804 & 50 & 001 \\
7.761 & 11.380 & 14 & 010 \\
7.870 & 11.224 & 23 & 011 \\
9.972 & 8.862 & 7 & 002 \\
10.406 & 8.494 & 54 & $0-11$ \\
10.650 & 8.299 & 100 & 012 \\
14.386 & 6.151 & 20 & $0-12$ \\
14.890 & 5.944 & 27 & 021 \\
15.577 & 5.684 & 19 & 020 \\
16.430 & 5.390 & 4 & $1-12$ \\
16.826 & 5.264 & 7 & $1-1-1$ \\
17.763 & 4.989 & 4 & $0-21$ \\
18.132 & 4.888 & 10 & 023 \\
18.912 & 4.688 & 65 & $0-13$ \\
19.208 & 4.617 & 16 & $1-13$ \\
19.455 & 4.558 & 5 & $1-21$ \\
19.784 & 4.483 & 8 & $1-1-2$ \\
20.279 & 4.375 & 14 & 104 \\
20.640 & 4.299 & 12 & $10-2$ \\
20.752 & 4.276 & 14 & 114 \\
21.467 & 4.136 & 23 & 024 \\
21.770 & 4.079 & 10 & $1-2-2$ \\
\hline
\end{tabular}

Table 15. Assignment of Eltrombopag free acid polymorphs and solvates characterized in this work, using, when possible, the original labeling, with the inclusion of the newly discovered features.

\begin{tabular}{|c|c|c|c|c|}
\hline Form & Solvent & Unit Cell & Comments on Original Data & Ref. \\
\hline I & None & Indexed & & [11] \\
\hline V & $\mathrm{THF} / \mathrm{H}_{2} \mathrm{O}$ & & Mixture of $\mathrm{THF} / \mathrm{H}_{2} \mathrm{O}$ solvates & [11] \\
\hline VI & $\mathrm{H}_{2} \mathrm{O}$ & Indexed & & [11] \\
\hline VII & None & Indexed & & [11] \\
\hline IX & THF & Indexed & & [11] \\
\hline$x$ & $2 \times \mathrm{DMSO}$ & Indexed & & [11] \\
\hline Xa & $1 \times$ DMSO & Indexed & & [11] \\
\hline XI & None & & Mixture rich in Form I & [11] \\
\hline XII & None & Indexed & & [11] \\
\hline XIV & EtOAc & Indexed & & [11] \\
\hline$X V$ & DMSO & & Mixture rich in Form 1x DMSO & [11] \\
\hline Z & DMF & & $=\mathrm{DMF}$ solvate & [12] \\
\hline DMF solvate & DMF & Indexed & & [12] \\
\hline DMA solvate & DMA & Indexed & & [12] \\
\hline NMP2 solvate & $2 \times \mathrm{NMP}$ & Indexed & & [12] \\
\hline NMP1 solvate & $1 \times \mathrm{NMP}$ & Indexed & & [12] \\
\hline $\mathrm{H} 1$ & DMF & & $=\mathrm{DMF}$ solvate & [13] \\
\hline
\end{tabular}

\subsection{Preparation and Characterization of the Unsolvated Forms of Eltrombopag}

Form I was easily reproduced by following the reported synthetic procedure [11]. As shown in Figure 1a, its diffraction peaks were relatively broad, and of markedly variable size and shape. Once cell refinement was performed, anisotropic particle sizes with average diameter near $50 \mathrm{~nm}$ were found. By applying slight modifications to the synthesis or thermal annealing, we could not improve this limited crystallinity. The fortunate isolation of a better crystallized material (ideally, Form XI), with the same powder diffraction pattern as Form I and sharper peaks, enabled the correct cell determination of a triclinic cell of a highly anisotropic shape. Looking at the proposed cell parameters, the classical dominant 
zone effect [14] was here present, weakening, to some (minor) extent, the accuracy of the evaluation of the very short real-space axis, falling slightly above $4 \AA$.

Form VII (Figure 1b) was prepared while heating, in the diffractometer cradle, powders of a mixture predominantly attributed to Form V (a tetrahydrofurane, THF, solvate); polyphasic in nature at room temperature, this mixture progressively evolved to the unsolvated Form VII through the intermediacy of another crystal phase (Form VI, later discussed). Obtained by high temperature treatment (above $180^{\circ} \mathrm{C}$ ), this material, after being cooled at room temperature, showed relatively broad peaks (as Form I did), but with a more limited spread in widths (speaking for less anisotropic particles, of average size close to around $45 \mathrm{~nm}$ ).

Moreover, the already known Form XII was isolated following the reported synthetic procedure [11]. As shown in Figure 1c, its diffraction peaks were much narrower than those observed for Forms I and VII. For Form XII, particle sizes near $80 \mathrm{~nm}$ were found. Variable Temperature X-ray Diffraction (VTXRD) analysis, which did not show any desolvation process (in agreement with Thermogravimetry and Differential Scanning Calorimetry, TG/DSC, data), see Figure 2), confirmed that Form XII is a truly polymorphic form of Eltrombopag Forms I and VII, with a nearly identical molar volume, and, consequently, similar crystal density. The similarity of the strength of the intermolecular interactions in all three unsolvated forms was further demonstrated by the incipient melting/decomposition temperatures falling in the narrow $246-249^{\circ} \mathrm{C}$ range.

All these unsolvated polymorphs are indefinitely stable, and did not show thermally induced solid-solid phase transitions from one to the other, as if highly reconstructive (and not displacive) molecular movements were necessary to cause polymorphic conversions. This is corroborated both by VTXRD and DSC measurements, gathered in Figure 2 for Forms I and XII. These VTXRD plots also indicated the most intense peaks above $22^{\circ} 2 \theta$, possessing $h \neq 0$ indices and related to the presence of a very short a axis (slightly above $4 \AA$ in both cases), shift upon heating to remarkably lower angles (i.e., to higher d-spacings) than $0 \mathrm{kl}$ peaks, in line with a plausibly shared molecular stacking in Forms I and XII, along which thermal expansion effects are predominant.

\subsection{Preparation and Characterization of the Solvated Forms of Eltrombopag: The Stoichiometric Phases}

The crystal forms discussed in this section, that is, Form IX (a THF solvate), Form $X$ (a dimethylsulfoxide-DMSO-solvate), and the $N, N^{\prime}$-dimethylformamide (DMF) and $N, N^{\prime}-$ dimethylacetamide (DMA) solvates (no Roman numerals being assigned to the latter), were prepared using the precipitation protocols illustrated in $[11,12]$. Highly crystalline phases have been recovered, the low-angle portions of their XRPD traces being shown in Figure 3. Tables 5-9 report the experimental peak positions and intensities and their assigned hkl indices.

Although distinct, Form IX and Form X show some similarity-beyond crystallizing in the same triclinic space group $(P-1)$, upon heating, they transformed into (distinct) intermediate phases (IXa and Xa, respectively), well before converting into unsolvated Form I (see Figure $4 \mathrm{a}, \mathrm{c}$ ). This process is easily understood for Form $X$, which possessed a 2:1 DMSO/Eltrombopag molecular ratio; in this case, partial desolvation (onset at around $114{ }^{\circ} \mathrm{C}$ ) led to a 1:1 (i.e., monosolvate) monoclinic structure, and only at $175^{\circ} \mathrm{C}$ was Form I quantitatively formed. VTXRD and TG/DSC analyses clearly showed the nature and extent of these processes (see Figure $4 b, d$ ). Separately prepared by heating powders of Form $\mathrm{X}$ (ex situ, at $150^{\circ} \mathrm{C}$ ), the $1: 1 \mathrm{DMSO}$ solvate was found to be stable at room temperature and provided an easily indexable XRPD trace (Figure 3c) and unit cell metrics apparently unrelated to that of Form X. 
Table 16. Synoptic collection of crystal data for the different phases of Eltrombopag.

\begin{tabular}{|c|c|c|c|c|c|c|c|c|c|c|c|c|c|}
\hline Form & I & $\mathbf{V}$ & VI & VII & IX & $X$ & $X a$ & XII & XIV & DMF & DMA & NMP2 & NMP1 \\
\hline Solvent & none & $\mathrm{THF}, \mathrm{H}_{2} \mathrm{O}$ & $\mathrm{H}_{2} \mathrm{O}$ & none & THF & $2 \times \mathrm{DMSO}$ & $1 \times \mathrm{DMSO}$ & none & EtOAc & DMF & DMA & $2 \times \mathrm{NMP}$ & $1 \times \mathrm{NMP}$ \\
\hline $\mathrm{Fw}, \mathrm{g} \mathrm{mol}^{-1}$ & 442.51 & 532.65 & 460.53 & 442.51 & 514.63 & 598.81 & 520.66 & 442.51 & 530.63 & 515.62 & 529.65 & 640.81 & 541.66 \\
\hline System & Triclinic & Triclinic & Triclinic & Triclinic & Triclinic & Triclinic & Monoclinic & Triclinic & Triclinic & Monoclinic & Triclinic & Monoclinic & Triclinic \\
\hline Space Group & $P-1$ & P-1 & $P-1$ & P-1 & $P-1$ & P-1 & $P 2_{1} / a$ & $P-1$ & P-1 & $P 2_{1} / n$ & P-1 & $P 2_{1} / c$ & P-1 \\
\hline$a(\AA)$ & 4.15 & 7.32 & 7.07 & 7.41 & 6.27 & 10.96 & 19.65 & 4.03 & 6.44 & 6.82 & 7.75 & 7.90 & 8.91 \\
\hline $\mathrm{b}(\AA)$ & 12.30 & 11.07 & 11.16 & 12.32 & 10.75 & 11.00 & 7.84 & 11.52 & 11.98 & 13.90 & 12.94 & 12.63 & 12.43 \\
\hline$c(\AA)$ & 22.26 & 17.09 & 15.77 & 12.93 & 20.58 & 13.63 & 17.48 & 24.29 & 19.46 & 27.58 & 13.62 & 33.66 & 13.96 \\
\hline$\alpha\left({ }^{\circ}\right)$ & 98.4 & 95.9 & 75.3 & 96.5 & 103.0 & 87.9 & 90 & 86.6 & 76.0 & 90 & 91.4 & 90 & 99.8 \\
\hline$\beta\left(^{\circ}\right)$ & 88.4 & 93.5 & 92.6 & 106.9 & 91.5 & 70.0 & 73.9 & 105.1 & 71.9 & 87.4 & 81.8 & 81.5 & 77.2 \\
\hline$\gamma\left({ }^{\circ}\right)$ & 89.2 & 98.7 & 77.9 & 105.1 & 96.2 & 79.5 & 90 & 89.0 & 97.6 & 90 & 89.7 & 90 & 111.2 \\
\hline$V\left(\AA^{3}\right)$ & 1123 & 1359 & 1171 & 1082 & 1341 & 1519 & 2586 & 1085 & 1350 & 2613 & 1351 & 3322 & 1400.1 \\
\hline $\mathrm{Z}, \mathrm{Z}^{\prime}$ & 2,1 & 2,1 & 2,1 & 2,1 & 2,1 & 2,1 & 4,1 & 2,1 & 2,1 & 4,1 & 2,1 & 4,1 & 2,1 \\
\hline $\mathrm{V} / \mathrm{Z}\left(\AA^{3}\right)$ & 561 & 680 & 585 & 541 & 670 & 759 & 646 & 543 & 675 & 653 & 675 & 830 & 700 \\
\hline $\mathrm{V} / \mathrm{Z}_{\text {calc }}{ }^{\mathrm{a}}\left(\AA^{3}\right)$ & 551 & 680 & 571 & 551 & 659 & 741 & 646 & 551 & 670 & 652 & 676 & 828 & 690 \\
\hline$\rho, \mathrm{g} \mathrm{cm}^{-3}$ & 1.333 & 1.300 & 1.335 & 1.333 & 1.297 & 1.342 & 1.338 & 1.333 & 1.314 & 1.314 & 1.301 & 1.285 & 1.304 \\
\hline Gof & 30.4 & 20.4 & 20.8 & 26.8 & 36.9 & 34.5 & 43.4 & 15.1 & 20.1 & 31.4 & 36.8 & 24.9 & 19.0 \\
\hline Onset $\mathrm{T},{ }^{\circ} \mathrm{C}$ & - & 80.8 & $160.6^{b}$ & - & 130.5 & 113.6 & 173.8 & - & 130.4 & 147.7 & 162.6 & 129.1 & - \\
\hline
\end{tabular}

a Values calculated from the statistical analysis approach described in Hoffmann [15]; ${ }^{\mathrm{b}}$ complex event. 
More intriguing is the formation of Form IXa, forming as an elusive crystalline phase while Form IX converted into Form I (see the VTXRD plot in Figure 4a where faint grey traces appear midway along the T coordinate (the $y$-axis). TG/DSC analysis of Form IX (see Figure $4 \mathrm{~b}$ ) indicated that the weak endothermic event occurring near $140{ }^{\circ} \mathrm{C}$ (onset at $130.5^{\circ} \mathrm{C}$ ) had a double component, with two closely overlapping peaks. Consequently, a XRPD trace of pure Form IXa could not be measured, nor were peak indexing and cell determination possible. From these data only, we could not assess the stoichiometry of this intangible phase, but, observing that the endothermic peak (DSC data) in the inset of Figure $4 \mathrm{~b}$ appeared to be slightly retarded with respect to the weight loss (TG data), we put forward the hypothesis that IXa is an unsolvated (and unstable) crystalline polymorph of Forms I, VII, and XII, easily transforming into Form I upon heating.

Of much simple interpretation were the VTXRD and DSC data obtained on the highly crystalline solvated phase labelled DMF and DMA solvates, both of 1:1 solvent/Eltrombopag stoichiometry. Upon heating, they uniquely showed solvent desolvation (onsets at 148 and $161{ }^{\circ} \mathrm{C}$, respectively), leading to Form I in a quantitative manner.

\subsection{Preparation and Characterization of the Solvated Forms of Eltrombopag: Crystal Phases of Uncertain Stoichiometry and/or Complex Characterization}

During the study of the crystal forms discussed in this section, and specifically Form V, Form XIV, and the $N$-methylpyrrolidone (NMP) solvate, we encountered unexpected difficulties, mostly related to the occurrence of polyphasic mixtures, some of which, upon heating, generated much more understandable XRD traces, attributed to rigorously monophasic materials.

Form V, prepared following the literature recipe [11], was indeed found to contain a polyphasic mixture, with a non-indexable XRPD pattern, as shown in Figure 5a. As demonstrated by the VTXRD plot illustrated in Figure 6a, it can be transformed, above $90{ }^{\circ} \mathrm{C}$, into a pure crystal phase, that is, Form VI, the cell symmetry and metrics of which are collected in Table 16. Its low-angle raw XRPD data were also inserted in Figure 5. As anticipated, further heating above $160{ }^{\circ} \mathrm{C}$ promoted the formation of the unsolvated phase Form VII, which could not be precipitated from solution nor obtained by thermally induced transformation of any of the other Eltrombopag crystal forms characterized in this work.

Following the published protocol [12], the so-called NMP solvate was also not easily isolated. The crude product recovered after filtration, indeed, was found to be a polyphasic mixture, dominated by an NMP-rich crystal phase (see the XRPD trace in Figure 5c). Selective peak choices enabled the determination of the monoclinic unit cell, with a volume corresponding to a 2:1 NMP/Eltrombopag molar ratio. Accordingly, we labeled this form NMP2, while that obtained after heating it above $110{ }^{\circ} \mathrm{C}$ was found to be a crystallographically pure material of 1:1 NMP/Eltrombopag ratio, herein identified as NMP1 (see Figure 5d). Interestingly, the XRPD data of NMP1 phase perfectly matched the NMP solvate phase originally characterized in [12]. Heating Form NMP1 above $180{ }^{\circ} \mathrm{C}$ generates Form I, as most (but not all) solvated crystal forms do.

Moreover, the XRPD trace of form XIV and its evolution with temperature (Figures 5e and 6e, respectively) proved to be a challenging task for their complete interpretation. At room temperature, an ethylacetate (EtOAc) solvate of triclinic symmetry was identified, with crystal data inserted in Table 16 . Near $90^{\circ} \mathrm{C}$, new peaks appeared, and the formation of a new phase, labeled as XIVa, was observed. The poor stability of this phase and the broad feature of its Bragg peaks prevented further metrical characterization. Above $120^{\circ} \mathrm{C}$, Form I was obtained, but, unexpectedly, a few sharp peaks of an additional and unknown crystal phase (here called XIVb, not indexable, as the number of independent reflections not overlapped with those of the predominant Form I was too low) were detected.

\subsection{Comparative Crystal Chemistry}

Eltrombopag free acid and its solvated forms crystallize in low symmetry crystal systems, mostly triclinic, and only in two cases as primitive monoclinic cells (Forms Xa and NMP1 solvate). In all cases, assuming that centrosymmetric space groups were present, 
Eltrombopag (pseudo)polymorphs showed a $Z=1$ value, with $Z$ being the number of formula units in the asymmetric portion of the unit cell. Moreover, thanks to this fortunate occurrence, peak indexing and cell determination proved to be a soluble task, even in the presence of broadened peaks or when only polyphasic samples were available.

While thermal methods (above all, TG) may properly suggest the nature, and extent, of the solvation process, the synthetic approaches we used strongly suggest which molecules are likely to be present. A further, very compelling set of observations (the molar volumes, that is, the $V_{\text {cell }} / Z$ values, included in Table 16 in $\AA^{3} / Z$ form, and easily transformed into $\mathrm{V}_{\mathrm{m}}$ in milliliters by using the $\mathrm{N}_{\mathrm{Av}} / 10^{24}$ conversion factor) also provided indirect measures of the crystal phase stoichiometries. Grounded on the well-known additivity of atomic volumes, valid in the absence of permanent porosity and crystal voids, Hofmann derived, from a statistical analysis of crystallographic databases, effective size effects for all atoms crystallizing in organics and in metallorganic compounds. [15] Accordingly, the experimentally derived molar volumes inserted in Table 16 were compared with those calculated using Hofmann's Table [15], under the constraint that the crystal phase stoichiometry was known. The high correlation among the two sets is depicted in Figure 7, where, if the correct estimate of the type and number of occluded solvent molecules is correct, a linear regression with a null intercept should provide a slope close to unity. Data shown in Figure 7 were linearly fit by the $\mathrm{y}=\mathrm{mx}$ straight line, resulting in $\mathrm{m}=1.007(4)$, with an $R^{2}$ value of 0.9998 . Notably, had wrong chemical formulae been inserted, such an excellent fit would not have been reached. This is particularly diriment in the case of Form $\mathrm{V}$ (a mixed solvent phase), of the monohydrated Form VI derived therefrom, and of the 2:1 solvent $/$ Eltrombopag crystal phases (solvent $=$ DMSO or NMP) with different, although still stoichiometric, solvent content.

\section{V/Z Correlation}

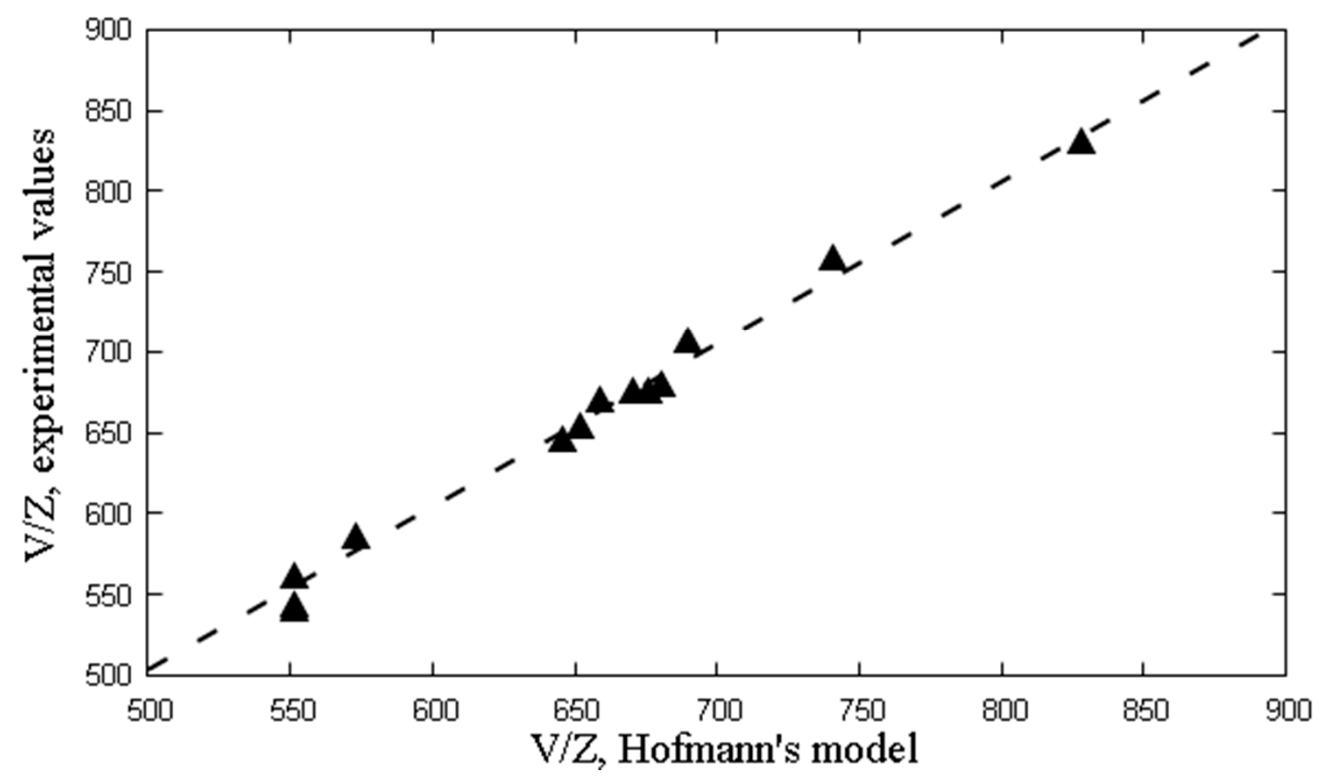

Figure 7. Molar volume correlation (values in $\AA^{3}$ ) for the different crystal phases indexed in this paper, showing how good quality XRPD data may be uses in a predictive manner to assess the exact solvent nature and stoichiometry within the large class of Eltrombopag solvates. The dashed line refers to the linear fit defined by $y=1.007 x$.

\section{Materials and Methods}

\subsection{Materials}

A $50 \mathrm{~g}$ industrial batch of Eltrombopag free acid was supplied by Chemessentia and characterized by solution ${ }^{1} \mathrm{H}-\mathrm{NMR}$ and ${ }^{13} \mathrm{C}-\mathrm{NMR}$ spectroscopy (NMR Avance 400 
spectrometer, Bruker, Billerica, MA, USA), as well as by XRPD. The crude product was found to consist of a physical mixture of Form I, Form V, and Form VI. Accordingly, ${ }^{1} \mathrm{H}-$ NMR showed additional peaks of the residual solvent (THF-water). ${ }^{1} \mathrm{H}-\mathrm{NMR}(400 \mathrm{MHz}$, DMSO-d $\left.{ }_{6}, 25{ }^{\circ} \mathrm{C}\right): \delta 13.78$ (bs, $\left.1 \mathrm{H}\right), 13.04$ (bs, $\left.1 \mathrm{H}\right), 9.71$ (bs, $\left.1 \mathrm{H}\right), 8.17(\mathrm{~s}, 1 \mathrm{H}), 7.99(\mathrm{~d}$, $J=7.6 \mathrm{~Hz}, 1 \mathrm{H}), 7.84(\mathrm{~d}, J=7.6 \mathrm{~Hz}, 1 \mathrm{H}), 7.75(\mathrm{~s}, 2 \mathrm{H}), 7.72-7.63(\mathrm{~m}, 2 \mathrm{H}), 7.30-7.15(\mathrm{~m}, 3 \mathrm{H})$, $2.37(\mathrm{~s}, 3 \mathrm{H}), 2.31(\mathrm{~s}, 3 \mathrm{H}), 2.26(\mathrm{~s}, 3 \mathrm{H}) .{ }^{13} \mathrm{C}-\mathrm{NMR}\left(100 \mathrm{MHz}, \mathrm{DMSO}-\mathrm{d}_{6}, 25{ }^{\circ} \mathrm{C}\right): \delta 167.22$, $156.86,147.78,142.52,137.65,136.77,135.76,133.35,132.78,132.19,131.04,130.61,129.88$, $129.76,128.89,128.32,128.26,127.09,121.93,118.88,115.32,113.95,19.63,18.82,11.52$.

Solvents (acetic acid (Sigma Aldrich, 99\%), acetone (Emplura, 99\%), anisole (Sigma Aldrich, 99\%), DMA (Merck, >98\%), DMF (Sigma Aldrich, 99.8\%), DMSO (Emplura, >98\%), ethanol (Emsure, 99\%), ethyl acetate (Carlo Erba, >99.8\%), methanol (Sigma Aldrich, 99.8\%), NMP (Sigma Aldrich, 99\%), THF (Sigma Aldrich, 99.9\%)) were used without purification.

\subsection{Preparation of the Different Eltrombopag Crystal Phases}

When, in the following sections, an unspecified form of Eltrombopag was used as a starting material, we refer to the crude product (the as-received laboratory batch described above in Section 2.1, consisting of a polyphasic mixture).

\subsubsection{Preparation of Eltrombopag Form I (Unsolvated)}

A total of $2.50 \mathrm{~g}$ of Eltrombopag was dissolved in $260 \mathrm{~mL}$ of glacial acetic acid ( $\geq 99 \%)$ in a four-neck $500 \mathrm{~mL}$ round bottom flask equipped with condenser and heated through an oil bath at the refluxing temperature of glacial acetic acid $\left(118{ }^{\circ} \mathrm{C}\right)$. Once a solution was obtained, the hot mixture was filtered on a porous septum using a water vacuum pump and left to crystallize overnight. The obtained product was filtered, collected, and dried at $35^{\circ} \mathrm{C}$ under vacuum. We obtained $1.627 \mathrm{~g}$ of a bright orange product.

\subsubsection{Preparation of Eltrombopag Form VII (Unsolvated)}

Eltrombopag Form V (200 mg) was deposited on a Petri dish and heated in an oven, initially at $110^{\circ} \mathrm{C}$ for $4 \mathrm{~h}$ and then at $160^{\circ} \mathrm{C}$ for $2 \mathrm{~h}$. The quantitatively recovered powder, after loss of the solvent, showed a significantly different diffraction trace than Form I and Form XII, witnessing the formation of a new unsolvated crystal phase.

\subsubsection{Preparation of Eltrombopag Form XI (Unsolvated)}

A total of $200 \mathrm{mg}$ of Eltrombopag was put in a $250 \mathrm{~mL}$ two-neck round bottom flask equipped with condenser, and $60 \mathrm{~mL}$ of acetone was added. The suspension was heated at the refluxing temperature until a clear solution was formed. The hot mixture was then filtered on a porous septum and the filtered solution was left at room temperature until complete evaporation of the solvent. Yield, $90 \mathrm{mg}$. XRPD clearly showed the identity of Form XI and Form I patterns, thus dismissing Form XI as being an independent crystal phase.

\subsubsection{Preparation of Eltrombopag Form XII (Unsolvated)}

Eltrombopag Form I $(20 \mathrm{mg})$ was dissolved in anisole $(6 \mathrm{~mL})$ using a two-neck $25 \mathrm{~mL}$ round bottom flask with heating and stirring until complete dissolution was achieved. The solution was then left at room temperature for 3 days. Agglomerated spherulites formed, filtered-off with the aid of gentle vacuum (yield: $12 \mathrm{mg}$ ).

\subsubsection{Preparation of Eltrombopag Form IX (THF Solvate)}

Eltrombopag Form I ( $20 \mathrm{mg}$ ) was dissolved in THF $(2 \mathrm{~mL})$ using a $3 \mathrm{~mL}$ cylindrical test flask with moderate heating and shaking. The mixture was then cooled and left at room temperature until complete evaporation of the solvent, leading to $20 \mathrm{mg}$ of an orange powder. 


\subsubsection{Preparation of Eltrombopag Form X (2:1: DMSO/Eltrombopag Solvate)}

Eltrombopag Form I $(20 \mathrm{mg})$ was dissolved in DMSO $(2 \mathrm{~mL})$ using a $3 \mathrm{~mL}$ cylindrical test flask with moderate heating and shaking. The mixture was then cooled and left at room temperature until a shiny orange powder was formed, later collected by filtration (12 mg).

\subsubsection{Preparation of Eltrombopag Form Xa (1:1 DMSO Solvate)}

The new Eltrombopag Form Xa was detected during a thermodiffractometric analysis of Form X. Indeed, heating Form $\mathrm{X}$ at around $140{ }^{\circ} \mathrm{C}$ quantitatively generates a still unknown crystal phase (Form Xa, in the following). Form Xa, stable after cooling down to room temperature, was then easily recovered and analyzed.

\subsubsection{Preparation of Eltrombopag DMF Solvate}

A total of $300 \mathrm{mg}$ of Eltrombopag and $1.05 \mathrm{~mL}$ of $\mathrm{N}, \mathrm{N}$-dimethylformamide were placed in a two-neck round bottom flask $(10 \mathrm{~mL})$ equipped with a condenser, and the suspension was heated via an oil bath at $70{ }^{\circ} \mathrm{C}$ under vigorous stirring for $30 \mathrm{~min}$. Then, $1.05 \mathrm{~mL}$ of absolute ethanol was added, and the mixture was stirred for 30 additional min, maintaining the working temperature constant $\left(70^{\circ} \mathrm{C}\right)$; during this time, the formation of a solid precipitate was observed. The mixture was then cooled to room temperature and kept under stirring for additional $15 \mathrm{~h}$. The obtained solid was filtered, washed with absolute ethanol, and dried in air, leading to the recovery of $266 \mathrm{mg}$ of a light orange powder.

\subsubsection{Preparation of Eltrombopag DMA Solvate}

A total of $200 \mathrm{mg}$ of Eltrombopag and $0.7 \mathrm{~mL}$ of $\mathrm{N}, \mathrm{N}$-dimethylacetamide were placed in a two-neck round bottom flask $(10 \mathrm{~mL})$ equipped with a condenser and heated via an oil bath until a clear solution at $80^{\circ} \mathrm{C}$ formed. The mixture was stirred at this temperature for $30 \mathrm{~min}$. Subsequently, $0.7 \mathrm{~mL}$ of absolute ethanol was added and the mixture was stirred for 30 additional min, maintaining the working temperature constant $\left(80^{\circ} \mathrm{C}\right)$; during this time, the formation of a solid precipitate was observed. The mixture was then cooled to room temperature and kept under stirring for 1 additional hour. The obtained solid was filtered, washed with $5 \mathrm{~mL}$ of absolute ethanol, and dried in air, leading to the recovery of $120 \mathrm{mg}$ of a bright orange powder.

\subsubsection{Preparation of Eltrombopag NMP (NMP2 and NMP1) Solvates}

A total of $300 \mathrm{mg}$ of Eltrombopag and $1.2 \mathrm{~mL}$ of NMP were placed in a two-neck round bottom flask $(10 \mathrm{~mL})$ equipped with condenser and heated under vigorous stirring for about $30 \mathrm{~min}$ until a clear solution at $75^{\circ} \mathrm{C}$ was formed. Then, $1.8 \mathrm{~mL}$ of ethanol was added and the mixture was stirred for additional $30 \mathrm{~min}$, maintaining the working temperature constant at $75^{\circ} \mathrm{C}$. The mixture was then cooled to room temperature and stirred for 1 additional hour. The obtained precipitate was filtered, washed with $0.5 \mathrm{~mL}$ of ethanol, and dried under vacuum (yield: $295 \mathrm{mg}$ ), and then characterized as the NMP2 crystal phase, containing NMP/Eltrombopag in a 2:1 molar ratio. Heating of this phase at $130{ }^{\circ} \mathrm{C}$ during VTXRD measurements promoted the quantitative formation of a different NMP solvate, labeled as NMP1, with a 1:1 stoichiometric ratio.

\subsubsection{Preparation of Eltrombopag Form V}

To $3.0 \mathrm{~g}$ of Eltrombopag placed in a $100 \mathrm{~mL}$ two-neck round bottom flask equipped with condenser, we added $17 \mathrm{~mL}$ of THF. The mixture was heated at the refluxing temperature $\left(90^{\circ} \mathrm{C}\right)$ until a clear solution was obtained. Then, water $(17 \mathrm{~mL})$ was added dropwise at this temperature and the solution was later cooled to room temperature and left under vigorous stirring for 1 additional hour. The precipitate was filtered, washed with water, and dried for $2 \mathrm{~h}$ at $50{ }^{\circ} \mathrm{C}$. The product was freeze-dried at $0.05 \mathrm{mbar}$ and $-53^{\circ} \mathrm{C}$ in order to remove the residual intergrain (non-structural) water molecules. 


\subsubsection{Preparation of Eltrombopag Form VI}

Eltrombopag Form V (200 mg) was placed in an alumina crucible and heated, with a heating ramp rate of $10^{\circ} \mathrm{C} \mathrm{min}-1$, in a muffle furnace up to $110^{\circ} \mathrm{C}$ and kept at this temperature for $20 \mathrm{~min}$ before cooling to room temperature. Quantitative transformation from form V to form VI was observed ( $189 \mathrm{mg}$ of material were recovered, in line with a possible monohydrate formulation of Form VI).

\subsection{X-ray Powder Diffraction Characterization}

\subsubsection{Experimental Conditions}

All powdered samples were gently ground in an agate mortar and deposited in the hollow of a zero-background plate (a $0.2 \mathrm{~mm}$ deep insert in a mis-cut silicon monocrystal). Diffraction data were collected at room temperature using a Bruker D8 AXS Advance diffractometer, operating in $\theta: \theta$ mode, and equipped with a $1 \mathrm{D}$ position sensitive detector (Lynxeye). Ni-filtered $\mathrm{Cu}-\mathrm{K} \alpha_{1,2}$ radiation; generator setting: $40 \mathrm{kV}, 40 \mathrm{~mA}$; goniometer radius $=300 \mathrm{~mm} ; 2.5^{\circ}$ Soller slits in the primary beam, divergence slit $=0.5^{\circ}$. A custommade antiscatter steel knife was positioned about $2.0 \mathrm{~mm}$ above the sample to limit the presence of parasitic $\mathrm{X}$-rays entering the detector window. Data were acquired in the full $3-105^{\circ} 2 \theta$ range, in step scan mode: $\Delta 2 \theta=0.02^{\circ}$, counting time $15 \mathrm{~s}$, overall duration: around $21 \mathrm{~h}$.

\subsubsection{Variable Temperature X-ray Diffraction}

These experiments were performed on the same equipment used in the previous section, modified by the presence of a custom-made sample heater (provided by Officine Elettrotecniche di Tenno, Ponte Arche, Italy), operated by an external controller with a nominal thermal stability of $\pm 0.1^{\circ} \mathrm{C}$. As the thermocouple is at the bottom of the aluminum sample holder and the surface of the sample is in contact with the air, some thermal gradients are expected, with the set temperature being a few degrees higher than the average one. DSC temperatures (discussed below) are then much more reliable than the XRPD ones.

\subsubsection{Determination of Crystallographic Unit Cell and Space Group Symmetry}

Standard peak search, followed by profile fitting and the fundamental parameters approach [16], provided a starting list of around 20 well defined, low-angle peak positions, fed to the indexing routine of TOPAS-R [17]. Through the singular value decomposition approach [18], cell determination and reflection hkl indices were derived, together with the goodness-of-fit parameters, GOF [19], listed at the bottom of Table 16. Allowance for sample-displacement, transparency, and flat-plate aberration errors was given, in the form of a constant zero-error shift, valid for a small low-angle portion of the data. Space group symmetry was assessed, coupling density considerations and, where pertinent, the analysis of systematic absences.

\subsection{Thermal Analyses}

Simultaneous thermogravimetric and differential scanning calorimetric analyses were performed under nitrogen atmosphere, in the RT to $400{ }^{\circ} \mathrm{C}$ range on a STA 409 PC Luxx equipment (Netzsch, Selb, Germany), using sample weights in the 5-10 mg range and open alumina crucibles at a $10^{\circ} \mathrm{C} \mathrm{min}{ }^{-1}$ scan rate. DSC onset points and TG mass losses were determined by Proteus Software version 4.8.1 (Netzsch, Selb, Germany).

\section{Conclusions}

In this paper, we reported the controlled syntheses of Eltrombopag free acid in its pure form or in a number of distinct crystalline polymorphs and solvates. The complete cell determination and peak indexing processes from accurate X-ray powder diffraction measurements certified the absence of contaminants and made the proposed crystallographic features a sound basis for polymoph/impurity detection in a large number of solid forms of 
Eltrombopag obtained under different operational conditions. The stability of the different (pseudo)polymorphs upon heating and their desolvation paths were studied, leading to the discovery of new intermediate phases or even of unexpected (room temperature-stable) forms of the pristine unsolvated material (Form I). Work can be anticipated in the direction of determining the full crystal structures by structural laboratory powder diffraction methods, very much in the way recently performed by us (and others) on crystal phases of drugs with molecules of moderate complexity [20]. Indeed, although promising structureless methods for polymorph quantification have appeared [21], this additional valuable information may help in quantifying contaminants and even amorphous phases in complex mixtures once the complete structural models are available [22].

Author Contributions: Conceptualization, G.B. and N.M.; methodology, N.M.; investigation, M.Z. and V.M.A.; writing, N.M.; project administration, M.Z.; funding acquisition, G.B. All authors have read and agreed to the published version of the manuscript.

Funding: This research was funded by Chemessentia s.r.l. The APC was covered by University of Insubria.

Data Availability Statement: The data presented in this study are available on request from the corresponding author. The data are not publicly available due to industrial protection issues.

Acknowledgments: V.M.A. thanks Chemessentia s.r.l. for partially funding his post-doctoral research contract. The courtesy of Stefano Brenna, University of Insubria, for technical assistance is heartily acknowledged.

Conflicts of Interest: The authors declare no conflict of interest.

Sample Availability: Not available.

\section{References}

1. Eltrombopag effective for ITP. Inpharma Wkly. 2006, 1544, 8. [CrossRef]

2. Available online: http://www.accessdata.fda.gov/drugsatfda_docs/nda/2008/022291s000_SumR.pdf (accessed on 19 November 2020).

3. Blokdijk, G.J.; Eltrombopag, O. A Complete Guide; CreateSpace Independent Publishing Platform: Scotts Valley, CA, USA, 2018.

4. Liu, K.K.-C.; Sakya, S.M.; O’Donnell, C.J.; Flick, A.; Li, J. Synthetic approaches to the 2009 new drugs. Bioorganic Med. Chem. 2011, 19, 1136-1154. [CrossRef] [PubMed]

5. Aitipamula, S.; Banerjee, R.; Bansal, A.K.; Biradha, K.; Cheney, M.C.; Choudhury, A.R.; Desiraju, G.R.; Dikundwar, A.G.; Dubey, R.; Duggirala, N.; et al. Polymorphs, Salts, and Cocrystals: What's in a Name? Cryst. Growth Des. 2012, 12, $2147-2152$. [CrossRef]

6. Vioglio, P.C.; Chierotti, M.R.; Gobetto, R. Pharmaceutical aspects of salt and cocrystal forms of APIs and characterization challenges. Adv. Drug Deliv. Rev. 2017, 117, 86-110. [CrossRef] [PubMed]

7. Bernstein, J. Polymorphism of Molecular Crystals, IUCr Monographs on Crystallography, 2nd ed.; Oxford University Press: Oxford, UK, 2020; Volume 30.

8. Brittain, H.G. (Ed.) Polymorphism of Pharmaceutical Solids, 2nd ed.; CRC Press: Boca Raton, FL, USA, 2016.

9. Karpinski, P. Polymorphism of Active Pharmaceutical Ingredients. Chem. Eng. Technol. 2006, 29, 233-237. [CrossRef]

10. McGilveray, I.J. Generic Drug Product Development Bioequivalence Issues. J. Pharm. Pharm. Sci. 2008, 11, 63. [CrossRef]

11. TEVA Phamaceuticals USA, Inc. Polymorphs of Eltrombopag and Eltrombopag Salts and Processes for Preparation Thereof. Patent No. US 20100256212, 7 June 2011.

12. Cadila Healthcare Ltd. Processes for the Preparation of Eltrombopag and Pharmaceutically Acceptable Salts, Solvates and Intermediates Thereof. Patent Application No. WO 2015/111085, 27 January 2015.

13. Hetero Research Foundation. Crystalline Forms of Eltrombopag Free Acid. Patent No. US 10336706B2, 2 July 2011.

14. Louër, D.; Boultif, A. Some further considerations in powder diffraction pattern indexing with the dichotomy method. Powder Diffr. 2014, 29, S7-S12. [CrossRef]

15. Hofmann, D.W.M. Fast estimation of crystal densities. Acta Crystallogr. Sect. B Struct. Sci. 2002, 58, 489-493. [CrossRef] [PubMed]

16. Cheary, R.W.; Coelho, A. A fundamental parameters approach to X-ray line-profile fitting. J. Appl. Crystallogr. 1992, 25, 109-121. [CrossRef]

17. TOPAS-R; Version 3.0; Bruker AXS: Karlsruhe, Germany, 2005.

18. Coelho, A.A. Indexing of powder diffraction patterns by iterative use of singular value decomposition. J. Appl. Crystallogr. 2003, 36, 86-95. [CrossRef]

19. De Wolff, P.M. A simplified criterion for the reliability of a powder pattern indexing. J. Appl. Crystallogr. 2002, 1, 108-113. [CrossRef] 
20. Vladiskovic, C.; Masciocchi, N. Reversibly changing a painkiller structure: A hot topic for a cold case-Ibuprofen lysine salt. J. Pharm. Biomed. Anal. 2015, 107, 394-402. [CrossRef] [PubMed]

21. Scarlett, N.V.Y.; Madsen, I.C. Quantification of phases with partial or no known crystal structures. Powder Diffr. 2006, 21, 278-284. [CrossRef]

22. Gualtieri, A.F.; Gatta, G.D.; Arletti, R.; Artioli, G.; Ballirano, P.; Cruciani, G.; Guagliardi, A.; Malferrari, D.; Masciocchi, N.; Scardi, P. Quantitative phase analysis using the Rietveld method: Towards a procedure for checking the reliability and quality of the results. Period. Miner. 2019, 88, 147-151. 